\title{
Prediction of Mechanical Properties of Polymers with Various Force Fields
}

\author{
Gregory M. Odegard* \\ Michigan Technological University \\ 1400 Townsend Drive \\ Houghton, MI 49931 \\ Thomas C. Clancy ${ }^{\dagger}$ and Thomas S. Gates ${ }^{\ddagger}$ \\ NASA Langley Research Center \\ Hampton, VA 23662
}

\begin{abstract}
The effect of force field type on the predicted elastic properties of a polyimide is examined using a multiscale modeling technique. Molecular Dynamics simulations are used to predict the atomic structure and elastic properties of the polymer by subjecting a representative volume element of the material to bulk and shear finite deformations. The elastic properties of the polyimide are determined using three force fields: AMBER, OPLS-AA, and MM3. The predicted values of Young's modulus and shear modulus of the polyimide are compared with experimental values. The results indicate that the mechanical properties of the polyimide predicted with the OPLS-AA force field most closely matched those from experiment. The results also indicate that while the complexity of the force field does not have a significant effect on the accuracy of predicted properties, small differences in the force constants and the functional form of individual terms in the force fields determine the accuracy of the force field in predicting the elastic properties of the polyimide.
\end{abstract}

\section{Introduction}

Nanostructured materials have the potential to provide significant increases in stiffness-to-weight and strengthto-weight ratios relative to current materials used for aerospace structural applications. To facilitate the development of these materials for this purpose, constitutive relationships must be developed that predict the bulk mechanical properties of the materials as a function of the molecular structure.

One promising approach to accomplish this type of modeling over multiple length scales is to establish the use of molecular dynamics (MD) simulations to accurately predict performance at the atomistic level. MD simulations can be used to predict the equilibrated molecular structure of a material and the behavior of the molecular system when subjected to applied mechanical deformations. Many studies have focused on the modeling and simulation of polymers and polymer-based nanocomposites via MD techniques. ${ }^{1-14}$ These studies have demonstrated that MD techniques can be effectively used to predict both structure and properties of polymer-based material systems. An important factor in the accurate prediction of properties of material systems via MD is the selection of the atomic potential. Several simplified atomic potentials, or force fields, for organic-based systems have been developed in recent years that describe the interactions between bonded and non-bonded atoms. ${ }^{15-36}$ Each of these force fields has been characterized via experimental techniques and quantum computations and has their own set of unique parameters and functional forms. Even though it is expected that these different parameters and forms will affect the relationship between force field type and predicted mechanical properties, little is known about this cause-and-effect relationship.

The objective of the present paper is to use MD simulations with different force fields to predict elastic properties of a polymer system. The predicted values of elastic properties from each force field will be compared to

\footnotetext{
* Assistant Professor, Department of Mechanical Engineering - Engineering Mechanics, Senior Member, AIAA.

${ }^{\dagger}$ Staff Scientist, National Institute of Aerospace, Member, AIAA.

* Senior Materials Research Engineer, Mechanics of Structures and Materials Branch, Associate Fellow, AIAA.
} 
experimentally measured values. The polymer system is a polyimide from 3,3',4,4'-biphenyltetracarboxylic dianhydride (BPDA) and 1,3-bis(4-aminophenoxy)benzene (APB) monomers (Figure 1). ${ }^{37,38}$ The three force fields used are the AMBER, OPLS-AA, and MM3 force fields. From these comparisons, the most appropriate force field for the prediction of mechanical properties of polymer-based nanocomposite systems is determined.

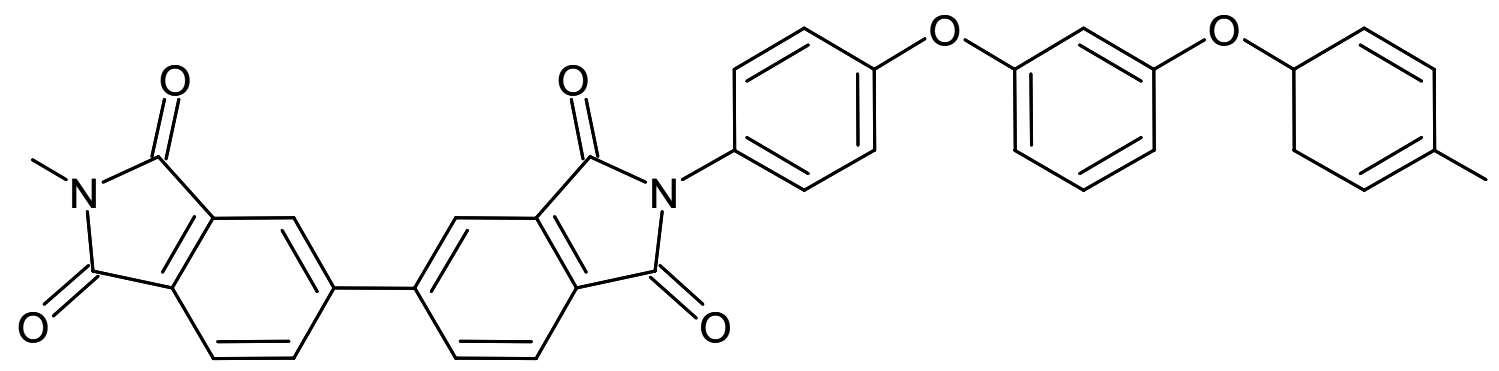

Figure 1. Schematic illustration of BPDA $(1,3,4)$ APB polyimide monomer unit

\section{Force Fields}

Three force fields were used in this study to simulate the polymer molecule deformation and compute the corresponding mechanical properties; the $\mathrm{AMBER}^{18,24-27}$ (without electrostatic interactions), OPLS-AA, ${ }^{28,29}$ and $\mathrm{MM} 3^{15,30-36}$ force fields. Each of the force fields has a unique functional form and set of force constants. The total potential energy of a simulated molecular system computed with the AMBER force field is

$$
\begin{aligned}
\Lambda_{\text {total }}= & \sum_{\text {stretch }} K_{r}\left(r-r_{e q}\right)^{2}+\sum_{\text {bend }} K_{\theta}\left(\theta-\theta_{e q}\right)^{2} \\
& +\sum_{\text {torsion }} \frac{V_{n}}{2}[1+\cos (n \phi-\zeta)]+\sum_{I<J} 4 \varepsilon_{I J}\left(\frac{\sigma_{I J}^{12}}{r_{I J}^{12}}-\frac{\sigma_{I J}{ }^{6}}{r_{I J}{ }^{6}}\right)
\end{aligned}
$$

where the summations are taken over all of the corresponding interactions in the molecular model; $K_{r}$ and $K_{\theta}$ are the bond-stretching and bond-angle bending force constants, respectively; $r$ and $r_{e q}$ are the bond length and equilibrium bond length, respectively; $\theta$ and $\theta_{e q}$ are the bond angle and equilibrium bond angle, respectively; $V_{n} / 2, \zeta, n$, and $\phi$ are the torsion magnitude, phase offset, periodicity of the torsion, and the torsion angle, respectively; and $\varepsilon_{I J}, r_{I J}$, and $\sigma_{I J}$ are van der Waals well depth, non-bonded distance between atoms $I$ and $J$, and the equilibrium distance between atoms $I$ and $J$, respectively.

The total potential energy of the molecular model computed with the OPLS-AA force field is

$$
\Lambda_{\text {total }}=\Lambda_{\text {stretch }}+\Lambda_{\text {bend }}+\Lambda_{\text {torsion }}+\Lambda_{\text {nb }}
$$

where

$$
\begin{gathered}
\Lambda_{\text {stretch }}=\sum_{\text {stretch }} K_{r}\left(r-r_{\text {eq }}\right)^{2} \\
\Lambda_{\text {bend }}=\sum_{\text {bend }} K_{\theta}\left(\theta-\theta_{\text {eq }}\right)^{2} \\
\Lambda_{\text {torsion }}=\sum_{\text {torsion }}\left\{\frac{V_{1}}{2}[1+\cos (\phi+\zeta)]+\frac{V_{2}}{2}[1-\cos (2 \phi+2 \zeta)]+\frac{V_{3}}{2}[1+\cos (3 \phi+3 \zeta)]\right\} \\
\Lambda_{\mathrm{nb}}=\sum_{I<J}\left[\frac{q_{I} q_{J} \mathrm{e}^{2}}{r_{I J}}+4 \varepsilon_{I J}\left(\frac{\sigma_{I J}{ }^{12}}{r_{I J}{ }^{12}}-\frac{\sigma_{I J}{ }^{6}}{r_{I J}{ }^{6}}\right)\right]
\end{gathered}
$$


where $q_{I}$ is the partial charge of atom $I$, e is the elementary charge, and the remaining quantities are those already defined for the AMBER force field.

For the MM3 force field, the total potential energy is

$$
\Lambda_{\text {total }}=\Lambda_{\text {stretch }}+\Lambda_{\text {bend }}+\Lambda_{\text {torsion }}+\Lambda_{\text {stretch-bend }}+\Lambda_{\text {torsion-stretch }}+\Lambda_{\text {bend-bend }}+\Lambda_{\text {vdw }}+\Lambda_{\text {electrostatic }}+\Lambda_{\mathrm{nb}}
$$

where

$$
\begin{aligned}
& \Lambda_{\text {stretch }}=\sum_{\text {stretch }} 71.94 K_{r}\left(r-r_{e q}\right)^{2}\left[1-2.55\left(r-r_{e q}\right)+1.49\left(r-r_{e q}\right)^{2}\right]
\end{aligned}
$$

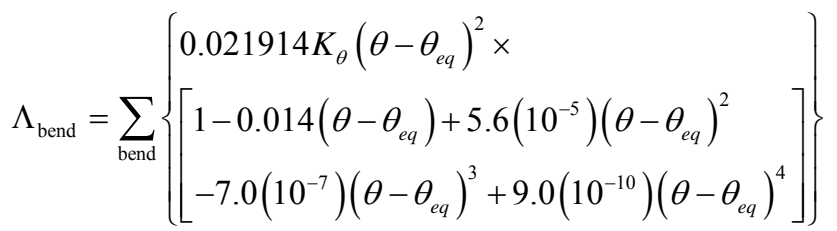

$$
\begin{aligned}
& \Lambda_{\text {torsion }}=\sum_{\text {torsion }}\left\{\frac{V_{1}}{2}[1+\cos (\phi)]+\frac{V_{2}}{2}[1-\cos (2 \phi)]+\frac{V_{3}}{2}[1+\cos (3 \phi)]\right\} \\
& \Lambda_{\text {stretch-bend }}=\sum_{\text {stretch-bend }} 2.51118 K_{r \theta}\left[\left(r-r_{e q}\right)+\left(r^{\prime}-r_{e q}^{\prime}\right)\right]\left(\theta-\theta_{e q}\right) \\
& \Lambda_{\text {torsion-stretch }}=\sum_{\text {torsion-stretch }} 11.995\left(\frac{K_{\phi r}}{2}\right)\left(r-r_{e q}\right)[1+\cos (3 \phi)] \\
& \Lambda_{\text {bend-bend }}=\sum_{\text {bend-bend }}\left[-0.021914 K_{\theta \theta^{\prime}}\left(\theta-\theta_{e q}\right)\left(\theta^{\prime}-\theta_{e q}^{\prime}\right)\right] \\
& \Lambda_{\mathrm{vdw}}=\sum_{I<J} \varepsilon_{I J}\left\{-2.25\left(\frac{\sigma_{I J}{ }^{6}}{r_{I J}{ }^{6}}\right)+1.84\left(10^{5}\right) \exp \left[-12.00\left(\frac{\sigma_{I J}}{r_{I J}}\right)\right]\right\}
\end{aligned}
$$

where $K_{r \theta}, K_{\phi r}$, and $K_{\theta \theta^{\prime}}$ are force constants; $r^{\prime}$ and $r_{e q}^{\prime}$ are the bond length and equilibrium bond length, respectively, of the adjacent covalent bond; and $\theta^{\prime}$ and $\theta_{e q}^{\prime}$ are the bond angle and equilibrium bond angle, respectively, of the adjacent bond angle. The energy contribution from electrostatic forces, $\Lambda_{\text {electrostatic }}$, is determined by either partial charges or dipole moments. The energies associated with all remaining non-bonded interactions, such as hydrogen bonding, is incorporated in $\Lambda_{\mathrm{nb}}$. Each of the force constants for these three force fields is unique for each force field and interacting atom types. For all three force fields, it was assumed that torsional force constants that were not defined in the respective literature references or by the simulation software ${ }^{39}$ were zerovalued.

\section{Equivalent-Continuum Modeling}

The nonlinear-elastic (hyperelastic) properties of the four material systems were determined using the Equivalent-Continuum Modeling method. ${ }^{8,9,40,41}$ This approach consisted of three steps. First, a representative volume elements (RVEs) of the molecular structure for each force field were chosen that accurately described the bulk structure of the material. Next, a constitutive law that described the behavior of the equivalent-continuum model was established. Finally, the energies of deformation of the two models were equated under identical sets of boundary conditions to determine each of the material parameters in the constitutive equation. Each of these steps is described in detail below.

\section{A. Representative Volume Element}

The RVEs of the molecular models were the equilibrium molecular structures of the polyimide for each force field determined using MD simulations. The RVE geometry of the molecular model selected was a cubic box. For this study, the molecular model of the polyimide was prepared with the aid of a reverse-mapping procedure from a 
coarse-grained model. ${ }^{42}$ This method is similar to those previously employed for multiscale polymer modeling. ${ }^{43,44}$ A brief summary of the method is given here with additional details presented elsewhere. ${ }^{46}$

For each polyimide molecule, a linked vector model was used to represent the rigid rings that comprise the polyimide backbone (Figure 2). The linked vectors followed the contour of the molecule. The parameters used for this model consisted of angular distributions between consecutive vectors and long-range forces between beads placed along the midpoint of each vector. These parameters were estimated from molecular dynamics (MD) simulation of the polyimide monomers with the CVFF force field. ${ }^{45}$ The centroids of the beads placed at the midpoint of each vector were the centers for interaction forces between non-adjacent beads along the chain of the polymer and between beads on different chains.

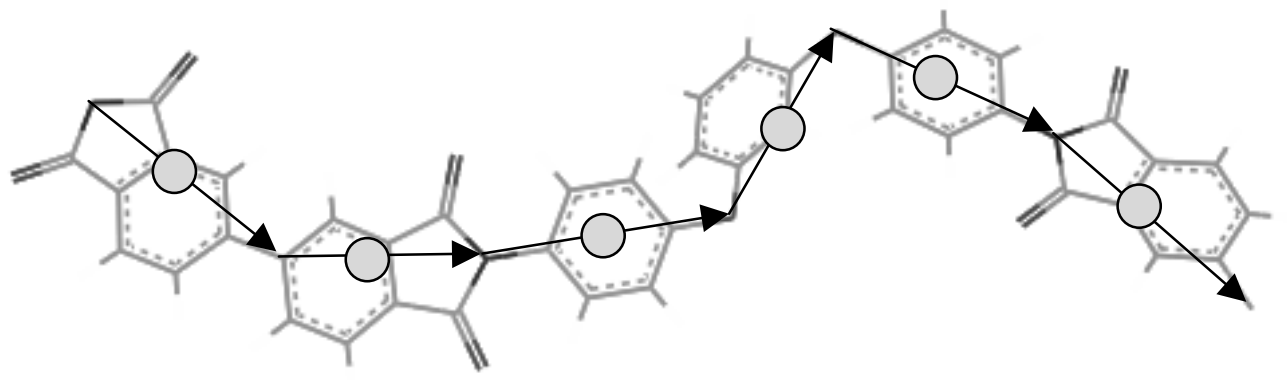

Figure 2. Depiction of the mapping of the atomistic polymer model to the coarse-grained linked vector model

Once the coarse-grained model for the single polyimide molecule was established, it was subsequently used to assemble the coarse-grained bulk model with multiple polymer molecules and the nanoparticle. The coarse-grained polymers were initially placed as random walk chains inside a simulation box (with periodic boundary conditions) close to their bulk density. In this initial placement, only the angular distributions between adjacent vectors along the chain were considered. Monte Carlo simulation was used to equilibrate the chains from their initial starting configuration. The bulk polymer model consisted of seven chains of polymers each composed of ten of the repeat units shown in Figure 1. This chain length is typical for MD simulations of polymers. The simulation box was a cube of side length $42 \AA$. The periodic box dimensions were chosen to allow the polymer to be close to the equilibrium bulk density. The simulation ran at $650 \mathrm{~K}$ until relaxation of the autocorrelation function of the end vectors was achieved and the average centers of mass were displaced a distance greater than the average radii of gyration squared. After sufficient equilibration with the coarse-grained Monte Carlo model, the chains were reverse-mapped by replacing the deleted atoms back into position along the vectors of the coarse-grained model.

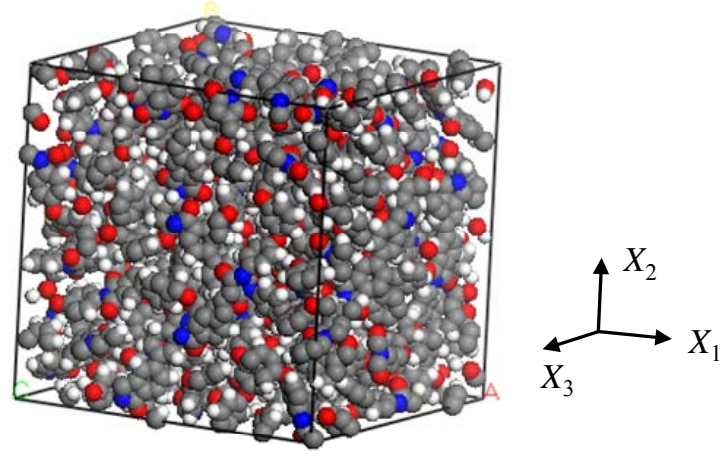

Figure 3. RVE of the polyimide material 
The resulting atomistic structures were subsequently minimized by the following procedure. A short energy minimization was applied to the structures. This was followed by an $n P T$ (constant number of atoms, pressure, and temperature) MD simulation for $200 \mathrm{ps}$ at $300 \mathrm{~K}$ and $1 \mathrm{~atm}$ using the AMBER, OPLS-AA, and MM3 force fields. This constant-pressure MD simulation allowed the atomistic structures to relax to the equilibrium density. The employed algorithm preserved the cubic structure of the simulation box while allowing the simulation box size to change. The final periodic boundary box sizes varied from $37.7 \AA$ to $47.2 \AA$ on a side depending on the force field used. After the $n P T$ MD simulations, the densities of the pure polyimide matrix was about $1.3 \mathrm{~g} / \mathrm{cm}^{3}$. This density is a reasonable value for polyimides. ${ }^{47}$ An example of a RVE of the polyimide is shown in Figure 3.

\section{B. Constitutive Equation}

For the computational simulation of polymer materials subjected to finite deformations, it is assumed that the strain energy function is associated with stress and strain tensors that are thermodynamic work conjugates in the balance of mechanical energy. In particular, the second Piola-Kirchhoff stress tensor is

$$
\mathbf{S}=\frac{\partial \Psi_{c}(\mathbf{E})}{\partial \mathbf{E}}
$$

where $\mathbf{E}$ is the Lagrangian strain tensor and $\Psi_{c}$ is the scalar strain-energy function of the equivalent continuum (defined per unit volume of the RVE in the reference configuration). The second Piola-Kirchhoff stress and Lagrangian strain tensors are henceforth referred to as the stress and strain tensors, respectively. Perhaps the simplest form of the strain energy function for isotropic hyperelastic materials is given by the classical Saint-Venant Kirchhoff model

$$
\Psi_{c}(\mathbf{E})=\frac{\lambda}{2}(\operatorname{tr} \mathbf{E})^{2}+\mu \operatorname{tr}\left(\mathbf{E}^{2}\right)
$$

where $\lambda$ and $\mu$ are the usual Lamé constants, $\operatorname{tr} \mathbf{E}$ is the trace of the tensor $\mathbf{E}$, and $\operatorname{tr}\left(\mathbf{E}^{2}\right)$ is the trace of tensor $\mathbf{E}^{2}$. It can be shown ${ }^{48}$

$$
\frac{\partial(\operatorname{tr} \mathbf{E})}{\partial \mathbf{E}}=\mathbf{I} \quad \frac{\partial\left(\operatorname{tr} \mathbf{E}^{2}\right)}{\partial \mathbf{E}}=2 \mathbf{E}
$$

where I is the second-order identity tensor. Substitution of Equations (16) and (17) into Equation (15) yields

$$
\mathbf{S}=\lambda \operatorname{tr}(\mathbf{E}) \mathbf{I}+2 \mu \mathbf{E}
$$

Equation (18) is the stress-strain relationship of the isotropic polymer material. This relationship describes the mechanical behavior of the equivalent-continuum model. At this point, however, the materials constants $\lambda$ and $\mu$ are unknown, and must be determined using on the molecular structure of the polymer. This is accomplished in the proceeding modeling step.

\section{Energy Equivalence}

The energies of deformation of the equivalent-continuum, $\Psi_{c}$, and molecular models, $\Psi_{m}$, were equated for identical sets of boundary conditions to determine the mechanical properties of the polyimide for each of the force fields. The molecular energy of deformation (potential energy) is

$$
\Psi_{m}=\frac{1}{V_{0}}\left(\Lambda_{\text {total }}-\Lambda_{\text {total }}^{0}\right)
$$


where $\Lambda_{\text {total }}^{0}$ and $\Lambda_{\text {total }}$ are the potential energies of the molecular model from Equations (1), (2), and (7) before and after deformation, respectively, and $V_{0}$ is the initial volume of the RVE. For finite deformations, the kinematic equations of motion that describe the deformation of the boundary of the RVE are generalized by

$$
\mathbf{x}=\mathbf{x}(\mathbf{X}, t)
$$

where $\mathbf{X}$ are the material coordinates (before deformation, defined in Figure 3), $\mathbf{x}$ are the spatial coordinates (after deformation), and $t$ is the time. The resulting strain that results from the deformation in Equation (20) is

$$
\mathbf{E}=\frac{1}{2}\left(\mathbf{F}^{T} \mathbf{F}-\mathbf{I}\right)
$$

where the superscript $T$ denotes a tensor transpose and $\mathbf{F}$ is the deformation gradient tensor whose components are given by

$$
F_{i j}=\frac{\partial x_{i}}{\partial X_{j}}
$$

Because the polymer materials are assumed to be isotropic, only two independent material parameters are required to completely describe the three-dimensional linear-elastic constitutive behavior described by Equation (18). A single set of deformations is required to determine each of the two independent parameters. The two independent parameters that were determined for these polymers where the bulk modulus, $\kappa$, and shear modulus, $\mu$. States of pure dilatation and pure shear were applied to the simulation box to determine $\kappa$ and $\mu$, respectively.

For the bulk deformations, the kinematic equations of motion (Equation (20)) become

$$
\begin{aligned}
& \mathbf{x}^{(1)}=\alpha_{1} \mathbf{X} \\
& \mathbf{x}^{(2)}=\alpha_{2} \mathbf{x}^{(1)} \\
& \mathbf{x}^{(3)}=\alpha_{3} \mathbf{x}^{(2)} \\
& \mathbf{x}^{(4)}=\alpha_{4} \mathbf{x}^{(3)}
\end{aligned}
$$

where $\alpha_{1}, \alpha_{2}, \alpha_{3}$, and $\alpha_{4}$ are scalar constants and the superscripts indicate the deformation step. The coordinates $\mathbf{x}^{(1)}$, $\mathbf{x}^{(2)}, \mathbf{x}^{(3)}$, and $\mathbf{x}^{(4)}$ correspond to bulk strains of $0.25 \%, 0.50 \%, 0.75 \%$, and $1.0 \%$, respectively. The relative deformation gradients (indicated by the apostrophe), which relate the deformation at a given strain level to those of the previous strain level are

$$
\begin{aligned}
& F_{i j}^{\prime}\left(\mathbf{x}^{(1)}\right)=F_{i j}\left(\mathbf{x}^{(1)}\right)=\frac{\partial x_{i}^{(1)}}{\partial X_{j}} \\
& F_{i j}^{\prime}\left(\mathbf{x}^{(2)}\right)=\frac{\partial x_{i}^{(2)}}{\partial x_{j}^{(1)}} \\
& F_{i j}^{\prime}\left(\mathbf{x}^{(3)}\right)=\frac{\partial x_{i}^{(3)}}{\partial x_{j}^{(2)}} \\
& F_{i j}^{\prime}\left(\mathbf{x}^{(4)}\right)=\frac{\partial x_{i}^{(4)}}{\partial x_{j}^{(3)}}
\end{aligned}
$$

Therefore, the deformation gradients that relate the coordinate for each strain level to those of the material coordinate system are 


$$
\begin{aligned}
& F_{i j}\left(\mathbf{x}^{(1)}\right)=\frac{\partial x_{i}^{(1)}}{\partial X_{j}} \\
& F_{i j}\left(\mathbf{x}^{(2)}\right)=\frac{\partial x_{i}^{(2)}}{\partial X_{j}}=F_{i m}^{\prime}\left(\mathbf{x}^{(2)}\right) F_{m j}\left(\mathbf{x}^{(1)}\right) \\
& F_{i j}\left(\mathbf{x}^{(3)}\right)=\frac{\partial x_{i}^{(3)}}{\partial X_{j}}=F_{i m}^{\prime}\left(\mathbf{x}^{(3)}\right) F_{m j}\left(\mathbf{x}^{(2)}\right) \\
& F_{i j}\left(\mathbf{x}^{(4)}\right)=\frac{\partial x_{i}^{(4)}}{\partial X_{j}}=F_{i m}^{\prime}\left(\mathbf{x}^{(4)}\right) F_{m j}\left(\mathbf{x}^{(3)}\right)
\end{aligned}
$$

Using Equations (22) - (25), the constants $\alpha_{1}, \alpha_{2}, \alpha_{3}$, and $\alpha_{4}$ where adjusted to achieve the exact desired strain levels of $0.25 \%, 0.5 \%, 0.75 \%$, and $1.0 \%$ in Equation (21). These values are listed in Table I.

Table I. Values of deformation parameters

\begin{tabular}{|c|c|c|}
\hline $\begin{array}{c}\text { Deformation } \\
\text { parameter }\end{array}$ & Value (unitless) & $\begin{array}{c}\text { Finite-valued strain } \\
\text { components }\end{array}$ \\
\hline$\alpha_{1}$ & 1.002497 & $E_{11}=E_{22}=E_{33}=0.25 \%$ \\
$\alpha_{2}$ & 1.002485 & $E_{11}=E_{22}=E_{33}=0.50 \%$ \\
$\alpha_{3}$ & 1.002472 & $E_{11}=E_{22}=E_{33}=0.75 \%$ \\
$\alpha_{4}$ & 1.002460 & $E_{11}=E_{22}=E_{33}=1.00 \%$ \\
$\beta_{1}$ & 0.001249 & $\gamma_{23}=\gamma_{13}=\gamma_{12}=0.25 \%$ \\
$\beta_{2}$ & 0.001246 & $\gamma_{23}=\gamma_{13}=\gamma_{12}=0.50 \%$ \\
$\beta_{3}$ & 0.001243 & $\gamma_{23}=\gamma_{13}=\gamma_{12}=0.75 \%$ \\
$\beta_{4}$ & 0.001240 & $\gamma_{23}=\gamma_{13}=\gamma_{12}=1.00 \%$ \\
\hline
\end{tabular}

For the pure shear simulations, the deformations of the four strain levels are

$$
\begin{aligned}
& x_{1}^{(1)}=\beta_{1}\left(X_{2}+X_{3}\right)+X_{1} \\
& x_{2}^{(1)}=\beta_{1}\left(X_{1}+X_{3}\right)+X_{2} \\
& x_{3}^{(1)}=\beta_{1}\left(X_{1}+X_{2}\right)+X_{3} \\
& x_{1}^{(k)}=\beta_{k}\left(x_{2}^{(k-1)}+x_{3}^{(k-1)}\right)+x_{1}^{(k-1)} \\
& x_{2}^{(k)}=\beta_{k}\left(x_{1}^{(k-1)}+x_{3}^{(k-1)}\right)+x_{2}^{(k-1)} \\
& x_{3}^{(k)}=\beta_{k}\left(x_{1}^{(k-1)}+x_{2}^{(k-1)}\right)+x_{3}^{(k-1)}
\end{aligned}
$$

where $\beta_{1}, \beta_{2}, \beta_{3}$, and $\beta_{4}$ are scalar constants and the superscripts $1,2,3$, and $4(k=2,3$, and 4$)$ correspond to pure shear strain levels of $\gamma_{23}=\gamma_{13}=\gamma_{12}=0.25 \%, 0.50 \%, 0.75 \%$, and $1.0 \%$, respectively $\left(\gamma_{i j}=2 E_{i j}\right.$ when $\left.i \neq j\right)$. Similar to the case of the pure bulk deformation, the constants $\beta_{1}, \beta_{2}, \beta_{3}$, and $\beta_{4}$ were adjusted such that these shear strains were achieved by using Equations (21) and (24) - (26). The values of the $\beta$ constants are listed in Table 1 .

The energies of deformation of the molecular models were determined using MD simulations. Both bulk and shear deformations were applied to the equilibrium molecular structures for each force field by deforming the MD simulation boxes and all of the atoms in the models according to the applied deformation field. An $n V T$ (constant number of atoms, volume, and temperature) simulation with periodic boundary conditions was subsequently used 
for each deformation to allow the box dimensions to remain fixed while the atoms were allowed to move into new equilibrium positions. The simulations were run up to $40 \mathrm{ps}$ at $298 \mathrm{~K}$, and were performed using the TINKER modeling package. ${ }^{39}$ The potential energies of deformation of the molecular models were averaged over the final 10 ps of each simulation, and the standard error of the energy fluctuations was recorded. A linear least-squares regression was performed for the potential energy of deformation over all four applied strain levels for each loading condition and force field. The elastic constants were subsequently determined using this data and Equation (16).

For the MD simulations, periodic boundary conditions were applied such that atoms were free to cross the boundary of the deformed and undeformed simulation cells. Atoms that crossed the boundary entered the simulation cells on the opposite side, as described in detail elsewhere. ${ }^{49}$ Therefore, none of the atoms in the MD simulations were kinematically over-constrained, as can occur in simulations of RVEs of heterogeneous material systems. ${ }^{50}$

\section{Results and Discussion}

Simulated values of the Young's and shear moduli of the polyimide are shown in Tables 2 and 3, respectively, for the three force fields. As a reference, an experimentally-obtained value of Young's modulus is shown in Table $2 .{ }^{37}$ The experimental shear modulus shown in Table 3 was obtained from the experimental Young's modulus and an assumed Poisson's ratio of 0.4. For each simulated value of Young's and shear modulus, the percentage difference with respect the experimental value is also shown in Tables 2 and 3 , respectively.

Table II. Predicted and Experimental Young's Modulus of Polyimide

\begin{tabular}{|c|c|c|}
\hline Method & Young's Modulus (GPa) & $\begin{array}{c}\text { \% difference from } \\
\text { experiment }\end{array}$ \\
\hline Experiment & $3.6^{*}$ & - \\
$\begin{array}{c}\text { Simulation } \\
\text { (AMBER force field) }\end{array}$ & 12.7 & $253 \%$ \\
$\begin{array}{c}\text { Simulation } \\
\text { (OPLS-AA force field) }\end{array}$ & 2.7 & $25 \%$ \\
\hline $\begin{array}{c}\text { Simulation } \\
\text { (MM3 force field) }\end{array}$ & 5.9 & $64 \%$ \\
\hline
\end{tabular}

* From reference [37]

From Table 2, it is clear that the force field which yielded a simulated Young's modulus that was closest to the experimental value was the OPLS-AA force field, with a value that was less than the experimental value. The simulations with the MM3 force field predicted a Young's modulus that was larger than the experimental value. The AMBER force field yielded a Young's modulus that significantly overestimated the experimental Young's modulus. From Table 3, the same trends exist for the simulated shear moduli. Again, The OPLS-AA force field predicted a shear modulus below the experimental value, whereas the shear modulus associated with the MM3 force field is larger than the experimental value. The shear modulus from the simulations with the AMBER force field significantly overestimated the experimental value.

Another important point can be drawn from the data in Tables 2 and 3. The functional forms of the AMBER and OPLS-AA force fields are very similar, as is evident from Eqns. (1)-(6). In addition, the force constants in these two force fields are also very similar. The functional forms of the AMBER and OPLS-AA are significantly more simple than that of the MM3 force field, Eqns (7)-(14). The MM3 force field contains many nonlinear and cross-interaction terms. Because of the similarity in the results of the OPLS-AA and MM3 force field simulations, and the significant overestimations of the AMBER simulations, it appears that small differences in functional form of the force field or values of the force constants have a significant effect on simulated elastic properties of this polyimide. The complexity of the MM3 force field doesn't appear to add significant accuracy for the specific simulations in this study. 
Table III. Predicted and Experimental Shear Modulus of Polyimide

\begin{tabular}{|c|c|c|}
\hline Method & Shear Modulus (GPa) & $\begin{array}{c}\text { \% difference from } \\
\text { experiment }\end{array}$ \\
\hline Experiment & $1.3 *$ & - \\
$\begin{array}{c}\text { Simulation } \\
\text { (AMBER force field) }\end{array}$ & 6.0 & $362 \%$ \\
$\begin{array}{c}\text { Simulation } \\
\text { (OPLS-AA force field) }\end{array}$ & 0.9 & $62 \%$ \\
\hline $\begin{array}{c}\text { Simulation } \\
\text { (MM3 force field) }\end{array}$ & 2.1 & $62 \%$ \\
\hline
\end{tabular}

* From reference [37] assuming $v=0.4$

The results of the AMBER and MM3 simulations in this study are similar to those of other studies in which the predicted values of mechanical properties are generally larger than the experimentally-determined properties. ${ }^{13,14}$ One explanation of this trend is that RVEs in molecular dynamics simulations represent a nearly perfect molecular structure. In the actual experimental test specimens, the material contains low volume fractions of air pockets, inclusions, and unreacted monomer. Therefore, from this perspective, it is expected that simulated mechanical properties should be larger than the experimental properties. Even though the OPLS-AA force field predicted mechanical properties that were closest to experimentally-determined properties, it may underestimate the mechanical properties of the specific RVE of the polyimide material that was modeled.

\section{Summary}

In this study, the effect of force field type on the predicted elastic properties of a polyimide has been examined. An equivalent-continuum modeling approach was used to predict the elastic properties based only on the atomic structure of the polyimide. Molecular Dynamics simulations were used to predict the atomic structure of the polymer and the corresponding potential energy changes when subjected to applied finite deformations. The energy changes were equated with the strain energy of the equivalent-continuum model, which was determined using a thermodynamic potential function and a finite deformation framework. As a result, the elastic properties of the polyimide were determined using three force fields: AMBER, OPLS-AA, and MM3. The predicted values of Young's modulus and shear modulus were compared with experimental values for the same polyimide.

The results of this study indicate that the mechanical properties of the polyimide predicted with the OPLS-AA force field most closely matched those from experiment. While the predicted properties using the MM3 force field were also reasonably close to experimental properties, those of the AMBER force field significantly overestimated the mechanical properties of the polymer. These results also indicate that the complexity of the force field does not have a significant effect on the accuracy of predicted properties. Apparently, small differences in the force constants and the functional form of individual terms in the force fields determine the accuracy of the force field in predicting the elastic properties of the polyimide. The results of the simulations with the MM3 and AMBER force fields exhibit similar trends those of other studies. For these force fields, the mechanical properties determined through the modeling overestimate those from experiment. This is most likely due to the imperfections that are present in bulk polymer specimens which are tested experimentally, which cannot be easily modeled on the molecular scale.

\section{Acknowledgments}

This research was sponsored by NASA Langley Research Center under grant NNL04AA85G and under the NASA cooperative agreement NCC-1-02043 with the National Institute of Aerospace. 


\section{References}

${ }^{1}$ Frankland, S. J. V., Caglar, A., Brenner, D. W. and Griebel, M., "Molecular Simulation of the Influence of Chemical Cross-Links on the Shear Strength of Carbon Nanotube-Polymer Interfaces," Journal of Physical Chemistry B, Vol. 106, 2002, pp. 3046-3048.

${ }^{2}$ Frankland, S. J. V., Harik, V. M., Odegard, G. M., Brenner, D. W. and Gates, T. S., "The Stress-Strain Behavior of Polymer-Nanotube Composites from Molecular Dynamics Simulation," Composites Science and Technology, Vol. 63, 2003, pp. 1655-1661.

${ }^{3}$ Griebel, M. and Hamaekers, J., "Molecular Dynamics Simulations of the Elastic Moduli of Polymer-Carbon Nanotube Composites," Computer Methods in Applied Mechanics and Engineering, Vol. 193, 2004, pp. 1773-1788.

${ }^{4} \mathrm{Hu}$, Y., Jang, I. and Sinnott, S. B., "Modification of Carbon Nanotube-Polystyrene Matrix Composites Through Polyatomic-Ion Beam Deposition: Prediction from Molecular Dynamics Simulations," Composites Science and Technology, Vol. 63, 2003, pp. 1663-1669.

${ }^{5} \mathrm{Hu}$, Y. H. and Sinnott, S. B., "Molecular Dynamics Simulations of Polyatomic-Ion Beam Deposition-Induced Chemical Modification of Carbon Nanotube/Polymer Composites," Journal of Materials Chemistry, Vol. 14, 2004, pp. 719-729.

${ }^{6}$ Liang, Z., Gou, J., Zhang, C., Wang, B. and Kramer, L., "Investigation of Molecular Interactions Between $(10,10)$ Single-Walled Nanotube and Epon 862 Resin/DETDA Curing Agent Molecules," Materials Science and Engineering A, Vol. 356, 2004, pp. 228-234.

${ }^{7}$ Lordi, V. and Yao, N., "Molecular Mechanics of Binding in Carbon-Nanotube-Polymer Composites," Journal of Materials Research, Vol. 15, 2000, pp. 2770-2779.

${ }^{8}$ Odegard, G. M., Clancy, T. C. and Gates, T. S., "Modeling the Mechanical Properties of nanoparticle/polymer composites," Polymer, Vol. 46, 2005, pp. 553-562.

${ }^{9}$ Odegard, G. M., Frankland, S. J. V. and Gates, T. S., "The Effect of Chemical Functionalization on Mechanical Properties of Nanotube/Polymer Composites," 44th AIAA/ASME/ASCE/AHS Structures, Structural Dynamics, and Materials Conference, Norfolk, VA, 2003.

${ }^{10}$ Odegard, G. M., Frankland, S. J. V., Herzog, M. N., Gates, T. S. and Fay, C. C., "Constitutive Modeling of Crosslinked Nanotube Materials," 45th AIAA/ASME/ASCE/AHS/ASC Structures, Structural Dynamics, and Materials Conference, Palm Springs, CA, 2004.

${ }^{11}$ Wei, C. Y., Srivastava, D. and Cho, K. J., "Thermal Expansion and Diffusion Coefficients of Carbon NanotubePolymer Composites," Nano Letters, Vol. 2, 2002, pp. 647-650.

${ }^{12}$ Sane, S. B., Cagin, T., Knauss, W. G. and Goddard, W. A., "Molecular Dynamics Simulations to Compute the Bulk Response of Ammorphous PMMA," Journal of Computer-Aided Materials Design, Vol. 8, 2002, pp. 87-106.

${ }^{13}$ Fan, C. F., Cagin, T., Chen, Z. M. and Smith, K. A., "Molecular Modeling of Polycarbonate. 1. Force Field, Static Structure, and Mechanical Properties," Macromolecules, Vol. 27, 1994, pp. 2383-2391.

${ }^{14}$ Fan, C. F. and Hsu, S. L., "Application of the Molecular Simulation Technique to Characterize the Structure and Properties of an Aromatic Polysulfone System. 2. Mechanical and Thermal Properties," Macromolecules, Vol. 25, 1992, pp. 266-270.

${ }^{15}$ Allinger, N. L., Yuh, Y. H. and Lii, J. H., "Molecular Mechanics. The MM3 Force Field for Hydrocarbons," Journal of the American Chemical Society, Vol. 111, 1989, pp. 8551-8566.

${ }^{16}$ Brooks, F. P., Bruccoleri, R. E., Olafson, B. D., States, D. J., Swaminathan, S. and Karplus, M., "CHARMM: A Program for Macomolecular Energy, Minimization, and Dynamics Calculations," Journal of Computational Chemistry, Vol. 4, 1983, pp. 187-217.

${ }^{17}$ Clark, M., Crammer, R. D. and Van Opdenhosch, N., "Validation of the General Purpose Tripose 5.2 Force Field," Journal of Computational Chemistry, Vol. 10, 1989, pp. 982-1012.

${ }^{18}$ Cornell, W. D., Cieplak, P., Bayly, C. I., Gould, I. R., Merz, K. M., Ferguson, D. M., Spellmeyer, D. C., Fox, T., Caldwell, J. W. and Kollman, P. A., "A Second Generation Force Field for the Simulation of Proteins, Nucleic Acids, and Organic Molecules," Journal of the American Chemical Society, Vol. 117, 1995, pp. 5179-5197.

${ }^{19}$ Foloppe, N. and MacKerell, A. D., "All-Atom Empirical Force Field for Nucleic Acids: I. Parameter Optimization Based on Small Molecule and Condensed Phase Macromolecular Target Data," Journal of Computational Chemistry, Vol. 21, 2000, pp. 86-104.

${ }^{20}$ Jorgensen, W. L., Madura, J. D. and Swenson, C. J., "Optimized Intermolecular Potential Functions for Liquid Hydrocarbons," Journal of the American Chemical Society, Vol. 106, 1984, pp. 6638-6646.

${ }^{21} \mathrm{Ott}, \mathrm{K} . \mathrm{H}$. and Meyer, B., "Parametrization of GROMOS Force Field for Oligosaccharides and Assessment of Efficiency of Molecular Dynamics Simulations," Journal of Computational Chemistry, Vol. 17, 1996, pp. 10681084. 
${ }^{22}$ Rappe, A. K. and Casewit, C. J., Molecular Mechanics Across Chemistry, University Science Books, Sausalito, CA, 1997.

${ }^{23}$ Sun, H., "COMPASS: An ab Initio Force-Field Optimized for Condensed-Phase Applications - Overview with Details on Alkane and Benzene Compounds," Journal of Physical Chemistry B, Vol. 102, 1998, pp. 7338-7364.

${ }^{24}$ Aqvist, J., "Ion-Water Interaction Potentials Derived from Free Energy Perturbation Simulations," Journal of Physical Chemistry, Vol. 94, 1990, pp. 8021-8024.

${ }^{25}$ Moyna, G., Williams, H. J., Nachman, R. J. and Scott, A. I., "Conformation in Solution and Dynamics of a Structurally Constrained Linear Insect Kinin Pentapeptide Analogue," Biopolymers, Vol. 49, 1999, pp. 403-413.

${ }^{26}$ Ross, W. S. and Hardin, C. C., "Ion-Induced Stabilization of the G-DNA Quadruplex: Free Energy Perturbation Studies," Journal of the American Chemical Society, Vol. 116, 1994, pp. 6070-6080.

${ }^{27}$ Wang, J., Cieplak, P. and Kollman, P. A., "How Well Does a Restrained Electrostatic Potential (RESP) Model Perform in Calculating Conformational Energies of Organic and Biological Molecules?" Journal of Computational Chemistry, Vol. 21, 2000, pp. 1049-1074.

${ }^{28}$ Jorgensen, W. L., Maxwell, D. S. and Tirado-Rives, J., "Development and Testing of the OPLS All-Atom Force Field on Conformational Energetics and Properties of Organic Liquids," Journal of the American Chemical Society, Vol. 117, 1996, pp. 11225-11236.

${ }^{29}$ Kaminsky, G. A., Friesner, R. A., Tirado-Rives, J. and Jorgensen, W. L., "Evaluation and Reparametrization of the OPLS-AA Force Field for Proteins via Comparison with Accurate Quantum Chemical Calculations on Peptides," Journal of Physical Chemistry B, Vol. 105, 2001, pp. 6474-6487.

${ }^{30}$ Allinger, N. L., Geise, H. H., Pyckhout, W., Paquette, L. A. and Gallucci, J. C., "Structures of Norbonane and Dodecahedrane by Molecular Mechanics Calculations (MM3), X-Ray, Crystallography, and Electron Diffraction," Journal of the American Chemical Society, Vol. 111, 1989, pp. 1106-1114.

${ }^{31}$ Allinger, N. L., Li, F. and Yan, L., "Molecular Mechanics. The MM3 Force Field for Alkenes," Journal of Computational Chemistry, Vol. 11, 1990, pp. 848-867.

${ }^{32}$ Allinger, N. L., Li, F., Yan, L. and Tai, J. C., "Molecular Mechanics (MM3) Calculations on Conjugated Hydrocarbons," Journal of Computational Chemistry, Vol. 11, 1990, pp. 868-895.

${ }^{33}$ Lii, J. H. and Allinger, N. L., "Molecular Mechanics. The MM3 Force Field for Hydrocarbons. 2. Vibrational Frequencies and Thermodynamics," Journal of the American Chemical Society, Vol. 111, 1989, pp. 8566-8575.

${ }^{34}$ Lii, J. H. and Allinger, N. L., "Molecular Mechanics. The MM3 Force Field for Hydrocarbons. 3. The van der Waals' Potentials and Crystal Data for Aliphatic and Aromatic Hydrocarbons," Journal of the American Chemical Society, Vol. 111, 1989, pp. 8576-8582.

${ }^{35}$ Lii, J. H. and Allinger, N. L., "Directional Hydrogen Bonding in the MM3 Force Field. I," Journal of Physical Organic Chemistry, Vol. 7, 1994, pp. 591-609.

${ }^{36}$ Lii, J. H. and Allinger, N. L., "Directional Hydrogen Bonding in the MM3 Force Field. II," Journal of Computational Chemistry, Vol. 19, 1998, pp. 1001-1016.

${ }^{37}$ Hergenrother, P. M., Watson, K. A., Smith, J. G., Connell, J. W. and Yokota, R., "Polyimides from 2,3,3',4'Biphenyltetracarboxylic Dianhydride and Aromatic Diamines," Polymer, Vol. 43, 2002, pp. 5077-5093.

${ }^{38}$ Srinivas, S., Caputo, F. E., Graham, M., Gardner, S., Davis, R. M., McGrath, J. E. and Wilkes, G. L., "Semicrystalline Polyimides Based on Controlled Molecular Weight Phthalimide End-Capped 1,3-Bis(4-

Aminophenoxy)Benzene and 3,3',4,4'-Biphenyltetracarboxylic Dianhydride: Synthesis, Crystallization, Melting, and Thermal Stability," Macromolecules, Vol. 30, 1997, pp. 1012-1022.

${ }^{39}$ TINKER - Software Tools for Molecular Design, Ver. 4.2, Washington University School of Medicine, St. Louis, MO, 2004.

${ }^{40}$ Odegard, G. M., Gates, T. S., Nicholson, L. M. and Wise, K. E., "Equivalent-Continuum Modeling of NanoStructured Materials," Composites Science and Technology, Vol. 62, 2002, pp. 1869-1880.

${ }^{41}$ Odegard, G. M., Gates, T. S., Wise, K. E., Park, C. and Siochi, E., "Constitutive Modeling of NanotubeReinforced Polymer Composites," Composites Science and Technology, Vol. 63, 2003, pp. 1671-1687.

${ }^{42}$ Baschnagel, J., Binder, K., Doruker, P., Gusev, A. A., Hahn, O., Kremer, K., Mattice, W. L., Muller-Plathe, F., Murat, M., Paul, W., Santos, S., Suter, U. W. and Tries, V., "Bridging the Gap Between Atomistic and CoarseGrained Models of Polymers: Status and Perspectives," Advances in Polymer Science, Vol. 152, 2000, pp. 41-156.

${ }^{43}$ Doruker, P. and Mattice, W. L., "A Second Generation of Mapping/Reverse Mapping of Coarse-Grained and Fully Atomistic Models of Polymer Melts," Macromolecular Theory and Simulations, Vol. 8, 1999, pp. 463-478.

${ }^{44}$ Tschop, W., Kremer, K., Batoulis, J., Burger, T. and Hahn, O., "Simulation of Polymer Melts. I. CoarseGraining Procedure for Polycarbonates," Acta Polymerica, Vol. 49, 1998, pp. 61-74.

${ }^{45}$ Materials Studio, Ver. 2.2, Accelrys Inc., San Diego, 2002. 
${ }^{46}$ Clancy, T. C. and Hinkley, J., "Coarse-Grained and Atomistic Modeling of Polyimides," NASA/TM-2004213030, 2004.

${ }^{47}$ Ashby, M. F. and Jones, D. R. H., Engineering Materials 1: An Introduction to Their Properties \& Applications, Butterworth-Heinemann, Oxford, UK, 1996.

${ }^{48}$ Holzapfel, G. A., Nonlinear Solid Mechanics: A Continuum Approach for Engineering, John Wiley \& Sons, Ltd., West Sussex, England, 2000.

${ }^{49}$ Haile, J. M., Molecular Dynamics Simulations: Elementary Methods, John Wiley \& Sons, Inc., New York, 1992.

${ }^{50}$ Sun, C. T. and Vaidya, R. S., "Prediction of Composite Properties from a Representative Volume Element," Composites Science and Technology, Vol. 56, 1996, pp. 171-179. 